\title{
LA INFLUENCIA DE EUROPA Y DE AMÉRICA LATINA EN LA EDUCACIÓN SUPERIOR DE ARGENTINA: UN RESUMEN DE LA EVALUACIÓN Y DE LA ACREDITACIÓNEN ESE PAÍS.
}

\author{
HELTON BARBOSA RODRIGUES ${ }^{1}$ \\ JACYGUARA COSTA PINTO ${ }^{2}$ \\ VANESSA DE SOUZA DA SILVA ${ }^{3}$
}

RESUMEN

Este artículoanalizala creación de la educación superior en Argentina y discute sobre su evaluación y acreditación, presentando todo un panorama de las convergencias entre Europa y América Latina, visto que, este proceso nace junto conlos cambios ideológicos ligados al ascenso del neoliberalismo económico. Este trabajo resalta la dimensión del espacio universitario y contribuye conlos demás trabajos académicos, ofreciendo elementos para la educación superior.

1 Mestre em Ciência da Educação, Licenciado Pleno em Geografia, Professor da rede pública estadual, atualmente trabalhando no Sistema Organizacional de Ensino Modular do Governo do Estado Amapá

2 Doutor em Ciência da Educação, Mestre em Ciência da Educação, Licenciado e Bacharel em História, Especialista Lato Sensu em Docência de Ensino Superior, Especialista Lato Sensu Em História cultura, Literatura Africana, afro-brasileira. Autor-participante dos livros: Mundo da Ciência: Coletânea de Artigos. São Paulo: Editora Anjos LTDA, 2019: Simpósio - Pós-Graduação em Educação. Macapá: Pró Reitoria de Pesquisa e Inovação -PROPESQ, 2015: Caderno de Projetos e Propostas Pedagógicas para a Implementação da Lei 10.639/03 no Estado do Amapá, 2014. Professor da rede pública estadual, atualmente trabalhando no Sistema Organizacional de Ensino Modular do Governo do Estado Amapá

${ }^{3}$ Mestrando em ciência da Educação, Especialista em Ensino Especial, Licenciada plena pedagogia, professora da rede pública estadual e Municipal, professora do Ensino Especial e educação infantil. 
PALABRAS - CLAVE: Europa, América Latina, Proceso de Boloña, Educación Superior, CONEAU, Evaluación, Acreditación.

\section{RESUMO}

Este artigo analisa a criação da educação superior na Argentina e discute sobre a sua avaliação e acreditação, apresentando todo um panorama das convergências entre a Europa e a América Latina, visto que, este processo nasce junto com as mudanças ideológicas ligadas à ascensão do neoliberalismo econômico. Esse trabalho ressalta a dimensão do espaço universitário e contribui com os demais trabalhos acadêmicos, oferecendo elementos para a educação superior.

PALAVRAS CHAVE: Europa, América Latina, Processo de Bolonha, Educação Superior, CONEAU, avaliação, acreditação.

\section{Introducción}

Las importantes transformaciones en el sistema capitalista mundial sucedieron al final del siglo XX (en todos los ámbitos: social, político, económico, tecnológico, cultural etc.) Una de ellas fue los cambios ocurridos en la educación superior, que pasó por un período de redefiniciones de sus funciones, vinculadas a los cambios impuestos por el proceso de globalizacióny regionalización del espacio mundial.

Los Sistemas Nacionales de Enseñanza Superior (SES) en América Latina son diversos, debido a que las políticas públicas varían de un país para otro, pues traenen su historia una gran influencia de sus colonizadores europeos sobre poderes ligados a la Iglesia y al Estado sin contar con influencias internas ocurridas en cada uno de ellos como cambios de regímenes políticos (Dictaduras militares), bien como la política neoliberal, que reduce la participación del Estado en la economía, y que forzó a las universidades a las prácticas de aproximacióna la demanda o racionalidad del mercado, ocasionando un impacto para esas instituciones, transformándolas en verdaderas ondas de expansión de matrículas que provocan cambios de alcance variado engrupos de desconcentración geográfica y de diferenciación institucional. En algunos casos, como en Argentina, la complacencia y evolución espontánea resultóen la inflación de las instituciones preexistente. 


\section{La influencia europea en el origen de la educación superior en América Latina.}

Según Durkheim (material digital), Boloñay Paris fueron las primeras universidades en Europa. Boloña, la más antigua, que data del año1088, caracterizada como la universidad de los estudiantes por su organización como naciones. La de Paris, la más importante, creada en el siglo XII, sirvió de modelo para otras instituciones. Oficializada en 1200, implantada dentro de los establecimientos religiosos, iglesias o monasterios, siendo sujeta areglamentos y disciplinas de la iglesia.

La influencia política de las universidades fue notable como primer ejemplo de organización puramente democrática. Los asuntos políticos, eclesiásticos y teológicos eran libremente debatidos, aunque se percibiera la inclinación para las clases privilegiadas, las universidades eran reconocidas en igualdad conla Iglesia, el Estado yla Nobleza (Monroe, 1979).

El siglo XVI introdujo en América Latina una educación superior aportada por los españoles, que fundaron la Universidad en México, Guatemala, Perú, Cuba, Argentina etc. El modelo europeo adoptado en la sociedad y en las universidades fue el francés, que ejercía fuerte influencia en Portugal y España.De esa manera, la educación superior estaba destinada solamente para la elite de los países latinos, como tambiénel acceso a los puestos políticos y burocráticos (Rosato, 2005).

Según Rosato, hacia el final del siglo XVIII, fueron creadas diecinueve universidades en América Latina y, posteriormente, otras treinta y una en el siglo XIX. Casi todos los países latinos americanos ya poseían una o más universidades, con excepción de Brasil.

Gradualmente, las universidades de América Latina dejaron de sufrir influencias de modelos europeos, como el francés, debido a que no conseguían conciliar la enseñanza profesional conla actividad científica. En el siglo XIX, América del Norte sufrió una gran expansiónen la enseñanza superior, como consecuencia la universidad latina no consiguió escapar de la influencia norte-americana, que avanzaba como un rollo comprensor sobre el continente.

Fue el modelo alemán del siglo XIX que estableció un padrón vinculado a la investigación científica conla enseñanza superior. En Francia, la actividad científica estuvo vinculada a los institutos independientes (...). En los Estados Unidos, que asimiló el modelo alemán, hubo innovación a nivel de formación de los científicos en los concursos de doctorado, formándolos para actividades universitarias y otras actividades externas, diferente de los doctorados europeos (Wanderley, 2005, p.20). 
El siglo XX, también llamado de siglo de la universidad, registró grandes avancesen los diversos campos sociales en varios países. La universidad se tornó una institución universal; generó una multiplicidad de modelos; se renovóen la gestión, estimulada por condiciones sociales emergentes; se flexibilizópara la formación, al lado de novas áreas de conocimiento; desarrolló másla educacióna distancia; crecióen el área politécnica, acompañando el intercambio dentro del continente; en algunos países ocurrió el crecimiento de la autonomía de la institución; y, finalmente, la investigación creció en las universidades, aumentando su credibilidad (Rosato , 2005).

En países en vías de desarrollo (Brasil, México y Argentina), la universidad presentó notable expansión, dejó de pertenecer a una pequeña parcela de estudiantes, se constituyó, muchas veces, en una esperanza de transformación delcuadro socioeconómico y pasó a ser colocada como centro de preocupaciones políticas (Rosato, 2005).

\subsection{Europa y el proceso de Boloña}

Europa también pasó por importantes transformaciones en la década de 1990. El avance de la globalización y la reestructuración del mercado hicieron que el plan de una unificación política y económica fuera concretado en 1992 conla creación de la Comunidad Europea (conocida más tarde como Unión Europea).

El objetivo era fortalecer la región entérminos políticos y económicos, de modo que ella pudiera preservar su papel principal frente a los cambios en el escenario mundial. Ese proceso, sin embargo, evocaba la necesidad de una serie de acciones que abarcaban desde el establecimiento de políticas comunes de comercio, como la política agrícola, hasta una reforma educativa superior, con el objetivo de establecer un padrón de normas comunes en los países miembros de la Unión Europea.

El conocido Proceso de Boloña, hoy incluyendo más de cuarenta países europeos, presentaba seis áreas de actuacióny buscaba ser un instrumento de coordinacióny de europeización de políticas (públicas) educativas (ANTUNES, 2007). Más que eso, su finalidad sería una sólida convergencia en la educación europea, a fin de que ésta respondiera adecuada y eficientemente a los problemas, oportunidades y desafíos gestionados por la globalización de la economía. 
De acuerdo con Riedoy Pereira (2009), los principales objetivos del Proceso de Boloña son: la promoción del sistema de Enseñanza Superior Europeo, inclusive a nivel mundial; la búsqueda de una armonización entre los sistemas universitarios, que facilitaría el reconocimiento de títulos de formación superior entre los países y ampliaría la oferta de profesionales altamente calificados disponibles en el mercado de trabajo; y "el estímulo a la movilidad de estudiantes, docentes y profesionales entre los países participantes, propiciando el intercambio de experiencias y conocimientos y que contribuiría para acelerar el desarrollo económico y cultural de cada país" (p. 61).

Para Bastos (2009), el Espacio Europeo de Educación Superior tiene como finalidad alcanzar los siguientes objetivos: a) aumentar la competitividad y la atracción a nivel internacional a la educación superior europea; b) mejorar la adaptación de la formación de los graduados europeos a las demandas del mercado de trabajo; d) desarrollarla movilidad interna y externa de alumnos. Una de las metas es establecer un sistema uniforme de créditos, organizado a partir de ciclos de estudios. La lógica del Proceso de Boloña es justamente ser un coadyuvante de las estrategias emprendidas por la Unión Europea en lo que se refiere a la educación superior.

A pesar de cierto optimismo relativo a la movilidad de los estudiantes, investigadores y docentes, la lógica de esa "armonización" entre los sistemas universitarios y de la uniformidad de los currículos, además del fuerte vínculo que se procura construir entre la formación y las demandas del mercado, son algunos aspectos que vienen siendo ampliamente debatidos en la comunidad europea y por estudiosos de otros países temerosos con la propagación del proceso de Boloña a los demás países.

Días Sobrinho (2009) establece dos críticas fundamentales a ese proceso: la primera se refiere a su excesiva centralización. La reforma fue estructurada de arriba a abajo, con poca participación de la comunidad universitaria y pasando por encima de la autonomía de las universidades. La segunda crítica se refierea las funciones esenciales de la educación superior. El autor establece que si el mercado determina la formación universitaria, imponiendo currículos de corta duración y enfocados al atendimiento de las necesidades laborales y de las características de los empleos del momento, muchos de la comunidad científica temen que la universidad pierda sus perspectivas de largo plazo y se aleje de las referencias sociales (p. 144). 
Además de eso, Días Sobrinho (2009) refuerza que esas relaciones de cooperacióncontienen un fuerte carácter utilitario. O sea, los intercambios poco tienen del sentido de la tradicional solidaridad universitaria o de una cooperación con el objetivo específico del desarrollo de la ciencia universal y desinteresada. El autor resalta que, sin que sea de forma absoluta, "en las relaciones entre sistemas, instituciones, profesores y estudiantes de los distintos países, lo que prevalece es el criterio de utilidad" (p. 144). Esa integración impone cierta homogenización del currículo y, a la vez, la pérdida de las funciones esenciales de la educación superior.

Prosiguiendo en esa dirección, Días Sobrinho (2009, p. 145) argumenta que los esfuerzos que están siendo hechos para aumentar la convergencia de la educación superior se tropiezan con tensiones y contradicciones bastante fuertes. De un lado, la creciente presión por aumentar la competitividad y aumentar los vínculos de la educación superior conel mercado ylas necesidades laborales. Por otro lado, la defensa de los valores académicos y de la función política de democratización. La visión de la educación como bien público orientado para la producción del bienestar de la populación es coherente con el refuerzo también explícito de los valores académicos, en contra posición a los intereses individualistas del mercado. Esta tensión no es particularmente limitada a Europa, pues, hace parte de las transformaciones de la sociedad global y de la educación superior de todas las partes, resguardadas las peculiaridades y diferencias de un país para otro.

La reestructuración del currículo tiene como discurso legitimador la posible "comparabilidad de formaciones diferentes para efectos de reconocimiento internacional y de movilidad, de transparencia de la mejoría efectiva de la cualidad de las formaciones" (BASTOS, 2009, p. 157). Para eso, la estructura académica curricular pasa a ser alterada y dividida en tres ciclos: el primero, básico, es con tres años de duración, seguido de una formación profesional de hasta tres años y un doctorado para aquellos que quieran dedicarse a la investigación.

Bastos (2009) apunta que ese sistema de ciclos ratifica un esquema perverso en el cual el mercado, en caso esté interesado en relaciones de trabajo más flexibles y disminución de los costos de mano de obra, contrata al estudiante que concluye apenas el primer ciclo de formación superior graduada. Eso auxiliará al mercado y a la promoción de la competitividad europea. Y, al mismo tiempo, el Estado disminuirá los encargos públicos con la educación superior, tornándose así, resultados prácticos de las políticas neoliberales. 
Esos cambios en el ámbito de la educación superior en Europa tiende a alterar completamente, según Bastos (2009), una de las grandes ventajas competitivas de Europa: la diversidad de estructuras de grados, diferenciación en los períodos de estudios, diversas formas de acceso, etc.

\section{Breve historia de la evolución política educativa superior en Argentina.}

Según Mollis (2008, p.518), la primer universidad de Argentina fue creada en 1613, la Universidad de Córdoba, fue protagonista del Movimiento de Reforma. Otras universidades tradicionales, tales como la Universidad de Buenos Aires yla Universidad de La Plata fueron creadas, respectivamente, en 1821 y 1905.

Fernando Lamarra (2005, p. 117), los orígenes de la educación superior en Argentina se remontan a la creación de un colegio jesuita en 1613, donde actualmente esla provincia de Córdoba. En 1622, se otorga a dicho colegiola calidad de universidad. La Universidad de Córdoba se nacionaliza.

El movimiento de Reforma ocurrido en la Universidad de Córdoba se constituyeen el primer gran cuestionamiento de la universidad en elámbito de América Latina y del Caribe, repercutiendo sobre todas las universidades argentinas y muchas latino-americanas. Sus principales propuestas fueron: a) autonomía universitaria; b) cogobierno de docentes yestudiantes; c) coexistencia de la universidad profesional con la científica; d) cátedras libres; e) renovación pedagógica; f) extensión universitaria; g) centralidad de los estudiantes y de los jóvenes como destinatarios y protagonistas de la universidad; h) solidaridad con el pueblo y los trabajadores; i) compromiso de la universidad conla transformación social; j) democratización de la educación superior, mediante su naturaleza gratuita (FERNÁNDEZ LAMARRA, 2005, p.118).

En Argentina, a partir de 1966, el golpe militar afectó especialmente a las universidades nacionales, que tuvieron su autonomía comprometida, generando acontecimientos “infames como la denominada 'Noche de los Bastones Largos', en la que fueron reprimidos y encarcelados estudiantesy docentes, originándose un primer éxodo de profesores e investigadores para otra instituciones y para el exterior" (FERNÁNDEZ LAMARRA, 2007, p. 162). En 1973 se estableció un nuevo gobierno democrático, depuesto en 1976 por otro golpe militar. La dictadura que persiguió, de forma sangrienta, docentes 
yestudiantes, generándose un segundo éxodo (muy grave y numeroso) de docentes, investigadores y estudiantes para otras instituciones y para el exterior del país.

La Argentina, como América Latina en general, también fue muy afectada por las políticas neoliberales. Desde 1995, los organismos internacionales de capital, tales como el Banco Mundial y el Banco Interamericano de Desarrollo, propusieron una agenda para la educación superior que incluyó: "la disminución de subsidios estatales para la educacióny la ciencia (...), la expansión de las instituciones y de la matrícula privada, la promulgación de una Ley de Educación Superior (...) y por último, la creación de órganos o agentes centrales para evaluar y acreditar las instituciones universitarias" (MOLLIS, 2008, p. 509-510).

La Ley de Educación Superior n. 24.521, del 7 de agosto de 1995, introduce cambios substantivos en lo que se refiere a los conceptos de autonomía, financiamiento y gobierno, en el sistema de educación superior argentino. Como ejemplo, autoriza a las instituciones universitarias a establecer el régimen de acceso, permanencia y salida de sus estudiantes de forma autónoma; autoriza a cada universidad que fije su propio régimen salarial docente y de administración de personal, asegurando el manejo descentralizado de los fondos; promueve la constitución de "sociedades, fundaciones u otras formas de asociación civil" destinadas a apoyar la gestión financiera y a facilitar las relaciones de las universidades y/o facultades conel medio; aumenta el número de representantes en los órganos colegiados de las instituciones. Hubo inclusive, a partir de esta Ley, el incremento de la pos-graduación pagada, como reflejo de la demanda por una mayor capacitacióny especialización de los estudiantes al término del ciclo universitario (MOLLIS, 2008, p. 512).

Según la autora, esta reforma se produjoen el contexto de las políticas neoliberales que "sustentaron la desregularización orientada por la libertad de los mercados en su correlación con la libertad otorgada a las universidades para cobrar tasas, pagar salarios diferenciados y crear regímenes de admisión (...) a diferencia de la tendencia del gobierno de controlar y 'evaluar' el desempeño de las instituciones universitarias” (2008, p.513-514). En ese sentido, la reforma de los años 1990, en Argentina, en semejanza con la ocurrida en Brasil en el mismo período, dio autonomía a las instituciones de educación superior para buscar fuentes de financiamiento diferentes al Estado, para diferenciarse en tipos de instituciones de calidad distinta, conel fortalecimiento de un proceso de evaluación centralizado en órganos como la Comisión Nacional de Evaluacióny Acreditación Universitaria (CONEAU), creada por la Ley n. $24.521 / 1995$.

El sistema de educación superior no-universitarioestá compuesto por institutos de formación docente y por institutos o escuelas especializadas de orientación técnica, 
profesionalizante. Cabe resaltar que, desde fines del siglo XIX y principios del siglo XX, fueron creados estos institutos. Los de formación docente para los niveles medio y superior tuvieron un gran desarrollo cuantitativo en la educación argentina. Por esto, hasta la actualidad, la mayor parte de los profesores es formado e nestos institutos no universitarios (FERNÁNDEZ LAMARRA, 2005, p. 118).

La diversificación de la educación superior argentina, de la misma forma que el caso brasileño, se intensifica a partir de los años 1990. De este modo, se fue configurando un conjunto de instituciones de educación superior altamente heterogéneo, con niveles de calidad también muy distintos.

En Argentina existe un equilibrio entre el número de instituciones públicas y privadas de educación superior, con pequeño predominio de estas últimas.

Con relacióna las matrículas, ocurre un fenómeno inverso al caso brasileño: $75 \%$ de ellas se encuentran en instituciones públicas y apenas $25 \%$ en privadas (GAZZOLA, 2008). Este dato nos remite al carácter predominantemente público y gratuito de la educación superior argentina.

En lo referente a la expansión del sistema de educación superior en su conjunto, esto es, el universitario y el no universitario, el primero domina elescenario, con 73,5\% de las matrículas contra 26,5\% de alumnos en nivel terciario no universitario (MOLLIS, 2008, p. 519). Esta tendencia del sistema argentino es altamente significativa cuando se compara con países como Brasil y México, que muestran un comportamiento inverso, con universidades públicas elitistas y una oferta masificada de enseñanza superior por las instituciones terciarias privadas, muchas de dudas a calidad, que no realizan investigación ni extensión.

Cabe resaltar, también, que la tasa de matrícula (TBM) en la educación superior, en Argentina, es de 54\%, contra apenas 24\% en Brasil (UNESCO, 2007 in: LÓPEZ SEGRERA, 2008, p. 274). Los datos seleccionados muestran el carácter más democrático y menos elitista de la educación superior argentina, cuando es comparada con la brasileña.

\section{La Comisión Nacional de Evaluacióny Acreditación Universitaria (CONEAU).}

Es un Organismo Público descentralizado que funciona en la jurisdicción del Ministerio de Educación Nacional, creado con la finalidad de contribuir parala mejora de la enseñanza universitaria, pues en el inicio de los años 2000, la educación pública fue subfinanciada, profundamente desmoralizada y debilitada para enfrentar el futuro de la nación. Desde 2003, la voluntad política para revertir esa situación puede ser vista en un indicador 
convincente: la inversión del Estado eneducación aumentó de 3,86\% del PIB e nese año para 6,47\% en 2011. Simultáneamente, la consolidación de CONEAU fue promovido como un elemento clave para mejorar la calidad de la enseñanza superior (investigación digital).

Es compuesto por doce miembros de la jerarquía académica y científica reconocida, con experiencia en gestión universitaria. Sirve a título personal, sin juzgamiento independiente y sin asumir la representación de cualquier institución. En CONEAU los miembros son nominados por el Ejecutivo Nacional, con base en la propuesta de las siguientes organizaciones y la cantidad indicada en cada caso:

- Tres por el Consejo Universitario Nacional.

- $\quad$ Uno por el Consejo de Rectores de las Universidades Privadas.

- Una por la Academia Nacional de Educación.

- $\quad$ Tres por el Senado de la Nación.

- $\quad$ Tres por la Cámara de Diputados.

- Uno por el Ministerio deEducación.

En CONEAU los miembros son nominados por cuatro años, con renovación parcial cada dos. Su presidente, vice-presidente y Secretario General son ejercidos entres años seleccionados a partir de ellos para una sociedad anual. Quincenalmente, la comisión se reúne en sesión plenaria. Para desempeñarlas funciones de evaluacióny acreditación de enseñanza superior Ley $\mathrm{n}^{\circ} 24.521$, atribuida a la comisión; está organizada en subcomisiones, con tareas agendadas y supervisan su equipo técnico permanente y presenta propuestas para las sesiones de las plenarias.

El equipo técnico que componela CONEAU está formado por universitarios profesionales disciplinares con diversos perfiles que se especializan en el desarrollo de procedimientos y técnicas de evaluación de las instituciones y carreras universitarias.

Actualmente ese tipo de equipo es organizado en las siguientes áreas:

- $\quad$ Dirección de Evaluación Externa y Proyectos Institucionales.

- $\quad$ Carrera Acreditación Dirección.

- $\quad$ Morada, Desarrollo, Planeacióny Relaciones Internacionales.

La organizaciónes completada por la Dirección de Administración, el Departamento de Registro de Peritos y Sistemas de Biblioteca y Área. 


\subsection{Proceso de Acreditación de Carrera}

El proceso de acreditación de carrera es realizado por un Organismo Público argentino funcional de la Secretaría de Políticas Universitarias del Ministerio de Educación, responsable por la evaluación de las universidades públicas y privadas y de la acreditación de sus alumnos de graduacióny pos-graduacióny sus correspondientes títulos. Él se tornó operativo en 1995 como parte de la Ley n ${ }^{\circ} 24.521$ de Enseñanza Superior (SLE) conducida durante el gobierno de Carlos Menem, en respuesta a las exigencias del Banco Mundial, integrando la Comisión de acreditación de Pos- graduación creada un año antes. La Resolución Ministerial n ${ }^{\circ}$ 160/11 del Ministerio da Educaciones la regla que rige la acreditación de los cursos de posgraduación en Argentina. Establece padrones mínimos de calidad a ser cumplidos por los programas de pos-graduación.

\section{Tipos de pos-graduación.}

De acuerdo con el título que conceda, y coherente con el artículo 39 de la Ley do Enseñanza Superior y de la Resolución Ministerial 160/11, Especialización, Maestría y Doctorado: los siguientes tipos son reconocidos carreras de pos-graduaciones.

- $\quad$ Especialización: que busca profundizar el dominio de un determinado asunto o área dentro de un campo profesional o diferentes profesiones.

- Maestría: busca proporcionar formación académica y/o profesional. profundiza el conocimiento teórico, metodológico, tecnológico, gerencial o artístico de acuerdo conel estado correspondiente a una disciplina o área profesional interdisciplinar de desarrollo de una o más profesiones.

- $\quad \mathrm{PhD}$ : procurala formación de pos-graduación que puede conseguir contribuciones originales en un área de conocimiento cuya universalidad debe buscar, dentro de un cuadro de excelencia académica por medio de entrenamiento que se concentra principalmente en torno de la investigación a partir del cual procurará hacer estas contribuciones originales.

\subsection{Resultado del Proceso de Acreditación}

En seis años la acreditación es hecha para las carreras que atienden el perfil establecido por las normas de acreditación. Una segunda posibilidad es conseguir la acreditación para tres años, en los casos en que el perfil pretendido no sea alcanzado, pero existen elementos suficientes para considerar que realmente desarrolla estrategias de mejora, 
cuyo impacto debe alcanzar el perfil requerido.

Su objetivo es promover las instituciones en relacióna la definición de políticas y mecanismos de garantía de calidad, fortaleciendo una cultura de evaluacióny control, incorporando las mejores prácticas de gestión institucional y enseñanza.

De acuerdo con el artículo 14 del Reglamento de la Ley 28.740: La acreditaciones el reconocimiento formal de la calidad otorgada por una institución o un programa educativo, concedida por el Estado, a través del Órgano Operador adecuado, como elbalance de evaluación externa emitido por una institución debidamente autorizada, de acuerdo con las normas actuales del organismo de evaluación. El SINEACE es una empresa especializada y en el ámbito del Ministerio deEducación de Perú, agencia técnica. La metodología utilizada para obtener la acreditación en calidad de enseñanza se basa en la realidad nacional. Trabaja con rigor técnico, objetividad y transparencia, y promueve la participación y coordinación con los diferentes actores y sectores públicos y privados.

\subsection{Comité de Pares}

Son un grupo de por lo menos tres disciplinas académicas y profesional de renombre, inscritos en el registro de peritos CONEAU que está en conformidad conel Consejo de Universidades, Instituciones Científicas y de profesionales, nacionales y provinciales. Esos especialistas tienen grados avanzados (especialistas, maestros, doctores) o formación equivalente, tal como ha sido demostrado por sus investigadores, profesores y profesionales. El tiende a preservar la diversidad de origen regional e institucional yla presencia de diferentes corrientes de especialistas extranjeros, científicas, filosóficas, metodológicas o tecnológicas.A su vez, la comisiónes un equipo técnico autónomo dividido en diferentes áreas que componen: Evaluación y Proyectos Institucionales, Acreditación y Licenciatura, Maestría, Desarrollo y Relaciones Institucionales, Gestión de Administración, Sistemas y Registros de Peritos y Biblioteca.

Las evaluaciones son realizadas por especialistas convocados ad hoc, organizados en consultoría, comités consultivos y comisiones de pares, con base en los cuales, CONEAU basa sus decisiones en cada caso. Los miembros de los comités de asesoría y pares se conducen de forma independiente, de acuerdo con un código de ética y tienen la capacidad de abstenerse de apertura de juzgamiento, si lo consideran adecuado. Su primer presidente fue

Emillo Fermin Mignone del movimiento activista de derechos humanos que surgió en Argentina durante la dictadura militar. 


\section{Consideraciones Generales}

La educación, a lo largo de la historia, siempre sirvió de aparato ideológico para la reproducción, el mantenimiento y/o transformación de la dinámica social. El neoliberalismo como ideología de actuación del Estado reforzó la idea de que sería por medio de la interacción de esas instituciones junto con el sector productivo que el progreso económico de los países se instauraría nuevamente. Entretanto, el sistema mundial de producción, fuertemente dependiente de la innovación tecnológica, precisando de mecanismos flexibles y de gran adaptación a la competitividad, reconoce que no basta la calificación profesional en el trabajo. Es necesario que esos IES (Institutos de Enseñanza Superior) comprendan la prerrogativa de estar siempre desarrollando "posibilidades" de aprendizaje y adaptarse frente a los cambios del conocimiento y las nuevas demandas del mercado y de la vida social (Días Sobrinho, 2005).

Los procesos de evaluación y acreditación enArgentina, en los años 90, incluyeron una comunidad académica que está cumpliendo un papel fundamental e nese proceso. El énfasis de la actividad desarrollada por la CONEAU está centrada en la dinámica interna de las instituciones, insistiendo para que sean las autoridades y la comunidad académica quienes definan las formas para garantizar y promover la calidad. La CONEAU enfatiza la autonomía de las instituciones como un aspecto fundamental para la mejoría de la calidad, por la responsabilidad que las instituciones de enseñanza superior asuman frente a la sociedad.

Estudiosos del asunto (Fernandez Lamarra n., 2007; WITT, H. 2005) prevén que, si la situación no fue controlada, probablemente, la validad los diplomas, incluyendo el contenido programático e, inclusive, las políticas educativas, en el futuro, no serán establecidas por los Gobiernos Nacionales, sino por las entidades supranacionales y estarán a merced de las presiones de los Estados más fuertes.

Para Buarque Apud Rosato (2005), la Universidad debe entender que existen dos tipos de futuro: el que es continuación linear del pasado, con nuevas respuestas para las mismas preguntas, dentro del momento paradigmático, y el que surge de la ruptura con el pasado, con la reformulación de la Organización social, con nuevas propuestas ideológicas, con preguntas diferentes. En esa perspectiva, la Universidad debe ser participante privilegiada de la gran aventura de construir al país. 


\section{Referencias Bibliográficas}

ANTUNES, Fátima (2009). O espaço europeu de ensino superior para uma nova ordem educacional? In: PEREIRA, Elezabethe Monteiro de Aguiar \& ALMEIDA, Maria de Lourdes Pinto de (Orgs.). Universidade contemporânea: Políticas do proceso de Boloña. Campinas. Mercado de Letras (PP.27-57). 167 p.

BASTOS, Carmem C. Barradas Correia. O proceso de Boloña no espaço europeu e a reforma universitária brasileira. In: PEREIRA, Elizabethe Monteiro deAguiar. Campinas: Mercado das Letras, 2009.p.155-164. [Links]

CONEAU. Lineamientos para La evaluación institucional. Buenos Aires: Nov. 1997.

DIAS SOBRINHO, José. Acreditación la educación superior. In: LOPEZ SEGRERA, Francisco; RIVAROLA, Domingo (Orgs.). La Universidad ante los desafíos Del siglo XXI. Asunção: Centro Paraguayo de Estudios Sociológicos, 2010.p. 110-294. [Links]

DURKHEIM, Emille. História de La educación y de evolución pedagógica en Francia. Material digital disponível no cd entregue no Seminário "La Universidade como Organización“.

GAZOLLA, Ana Lúcia. Panorama de La educación superior en América Latina y el Caribe. Cartagena de Indias: UNESCO/ IESALC, junho de 2008.

LAMARRA, Norberto Fernandéz (Orgs.). Educación superior. Convergencia entre América Latina y Europa. Caseros: Eduntref, p. 117-283, 2005.

_ Educación superior y Calidad em América Latina y Argentina. Los procesos de evaluación y acreditación. Caseros: Eduntref, 2007.

MOLLIS, Marcela. Identidades alteradas: de lãs universidades reformistas a las universidades de La reforma. In: BERNHEIM, Carlos Tunnermann (Ed.). La educación superior en América Latina y El Caribe: diez años después de La Conferencia Mundial de 1998. Cali: Iesal- Unesco, PUJ, 2008. [ Links ] 
MONROE, Paul. História da educação. São Paulo: Ed. Nacional, 1979.

RIEDO, Cássio Ricardo Fares; PEREIRA, Elizabethe Monteiro de Aguiar. O proceso de Boloña e suas consequiências na Itália. In: PEREIRA, Elizabethe Monteiro de Aguiar, Maria de Lourdes Pinto (Orgs.) Universidade Contemporânea. Políticas do proceso de Boloña. Campinas, SP: Mercado das Letras, 2009.p. 59- 80.

ROSATO, Ricardo. Universidade: nove siglos de História. Paso Fundo: UPF, 2005. UNESCO, 2007 In: LOPEZ SEGRERA, 2008, p. 274.

WANDERLEY, L.E.W. O que é Universidade? São Paulo: Brasiliense, 2005. 